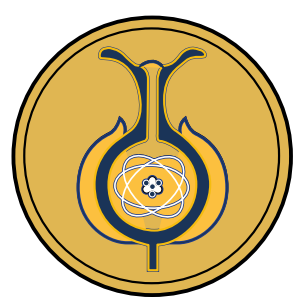

\title{
Conocimiento especializado del profesorado de matemática en formación inicial acerca de los polígonos
}

\author{
Specialized knowledge of polygons of math teachers in training
}

Conhecimento especializado de professores de matemática na formação inicial sobre poligonos

Elizabeth Advíncula-Clemente ${ }^{1}$, Marisel Beteta-Salas ${ }^{1}$, José León-Ríos ${ }^{1}$, Isabel Torres-Céspedes ${ }^{1}$, Miguel Montes ${ }^{2}$

Received: Dec/30/2020 • Accepted: Set/18/2021 • Published: Jan/31/2022

\section{Resumen (10)}

En este artículo nos centramos en comprender el conocimiento especializado que muestra una profesora de educación secundaria en formación, cuando enseña polígonos como parte de su práctica preprofesional en el último año de su carrera. Para explorar dicho conocimiento nos basamos en el modelo Mathematics Teacher's Specialised Knowledge (MTSK) creado por el grupo de investigación de la Universidad de Huelva, España. Utilizamos una metodología de investigación cualitativa y desde el paradigma interpretativo damos cuenta de un estudio de caso que permitirá identificar, describir y comprender el conocimiento geométrico de la maestra referente a los polígonos. La recogida de información se realizó a través de observación no participante, mediante grabaciones realizadas durante tres sesiones de clase con estudiantes de quinto grado de educación secundaria. Para la validación del análisis usamos la triangulación de expertos externos en el modelo MTSK. Los resultados evidencian el potencial que ofrece el MTSK como herramienta para profundizar en la caracterización y comprensión del conocimiento del profesorado, cuyo uso resultaría favorable en la formación inicial de docentes de educación secundaria, pues permite que el personal docente en formación inicial reflexione sobre la necesidad de tener un conocimiento matemático y didáctico sobre el contenido a enseñar. Los resultados muestran como el conocimiento del tema de polígonos se relaciona íntimamente con el conocimiento de la enseñanza de la matemática vinculado a este tema, así como al conocimiento de las características del aprendizaje de la matemática.

Palabras clave: Conocimiento especializado; docente en formación; educación secundaria; polígonos.

\footnotetext{
Elizabeth Advíncula-Clemente, $\$ eadvincula@pucp.edu.pe, (D: https://orcid.org/0000-0003-3941-3139

Marisel Beteta-Salas, \ mbeteta@ulima.edu.pe, (1D: https://orcid.org/0000-0003-01000-8517

José León-Ríos, \jleonr@ulima.edu.pe, D: https://orcid.org/0000-0002-3128-788X

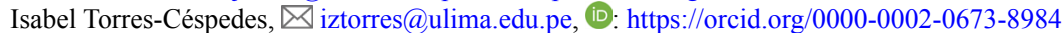

Miguel Montes, \miguel.montes@ddcc.uhu.es, (D: https://orcid.org/0000-0003-3181-0797

1 Instituto de Investigación Científica, Universidad de Lima, Lima, Perú.

2 Centro de investigación COIDESO de la Universidad de Huelva, Huelva, España.
} 


\section{Abstract (1D)}

The paper is aimed to understand the specialized knowledge shown by a secondary education teacher in training when teaching polygons as part of her pre-professional practice on her senior year. This exploration was based on the Mathematics Teacher's Specialized Knowledge (MTSK) model developed by a research group in the University of Huelva, Spain. The research methodology was qualitative. Based on an interpretive approach, a case study was used to identify, describe, and understand the student teacher's geometric knowledge of polygons. Information was collected through non-participant observation, using recordings of three sessions with fifth graders of secondary education. In order to validate the analysis, we used external expert triangulation in the MTSK model. The results show the potential offered by the MTSK model as a tool to deepen the characterization and understanding of teachers' knowledge, favoring the initial training of secondary education teachers by allowing them to reflect on the need to have a mathematical and didactic knowledge of the content to be taught. Results show how the knowledge of polygons is closely related to the knowledge of teaching mathematics linked to this subject, as well as to the knowledge of the characteristics of mathematics learning.

Keywords: specialized knowledge; teacher in training; secondary education; polygons.

\section{Resumo}

Neste artigo, focamos na compreensão do conhecimento especializado demonstrado por uma professora do ensino médio em formação ao ensinar polígonos como parte da prática pré-profissional em seu último ano acadêmico. Para explorar esse conhecimento, apoiamo-nos no modelo de Mathematics Teacher's Specialised Knowledge (MTSK)(Conhecimento Especializado do Professor de Matemática)criado pelo grupo de pesquisa da Universidade de Huelva, Espanha. Utilizamos uma metodologia de pesquisa qualitativa e, a partir do paradigma interpretativo, explicamos um estudo de caso que nos permitirá identificar, descrever e compreender o conhecimento geométrico da professora sobre polígonos. As informaç̃ões foram coletadas por meio de observação não participativa, mediante gravações feitas durante três aulas com alunos do quinto ano do ensino médio. Para a validação da análise, usamos a triangulação de especialistas externos no modelo MTSK. Os resultados mostram o potencial oferecido pelo MTSK como ferramenta para aprofundar na caracterização e compreensão do conhecimento dos docentes, cujo uso seria favorável na formação inicial de professores do ensino médio, pois permite àqueles em formação inicial refletir sobre a necessidade de ter um conhecimento matemático e didático sobre o conteúdo a ser ensinado. Os resultados mostram como o conhecimento do tema dos polígonos está intimamente relacionado ao conhecimento do ensino da matemática vinculado a esse tópico, bem como ao conhecimento das características da aprendizagem da matemática.

Palavras-chave: Conhecimento especializado; professores em formação; ensino médio; polígonos. 


\section{Introducción}

La formación de docentes de matemáticas es objeto de estudio desde diferentes ámbitos. Encontramos investigaciones que reportan que el personal docente evidencia una escasa preparación en aspectos matemáticos y didácticos. Por ejemplo, Díaz (2015) menciona que egresan con competencias matemáticas insuficientes y reproducen prácticas pedagógicas tradicionales, emulando el estilo con el que recibieron su formación. Asimismo, Osorio (2017) señala que en el Perú existe poca preparación en temas matemáticos y didácticos. Esta autora considera, como reto en la formación docente, aumentar la preparación en temas de matemática y aspectos didácticos de esta disciplina.

Coincidimos con investigaciones que señalan que el conocimiento profesional de docentes de matemáticas debe integrar el conocimiento disciplinar y el propio de la didáctica del contenido (Ball, Thames y Phelps, 2008; Shulman, 1986), asumiendo que dicho conocimiento debe ser especializado (Carrillo et al., 2018). Por tanto, consideramos fundamental asegurar la construcción tanto del conocimiento disciplinar como didáctico del profesorado en su formación inicial.

En el Perú, el Ministerio de Educación es el encargado de desarrollar políticas educativas que promuevan la formación de docentes de educación básica. Dado que nuestro trabajo se centra en docentes de educación secundaria en formación inicial, revisamos el Diseño Curricular Básico Nacional para la Carrera Profesional de Profesor de Educación Secundaria en la especialidad de Matemática (MINEDU, 2010), a fin de conocer el plan de estudios de la especialidad de Matemática; asimismo, revisamos el Currículo Nacional de la Educación Básica y el Programa Curricular de Educación Secundaria, documentos en los que, respecto a los polígonos, se señala que al egresar de la educación básica el estudiantado debe ser capaz de clasificar polígonos según sus propiedades, reconociendo la inclusión de una clase en otra, seleccionar, combinar y adaptar variadas estrategias, procedimientos y recursos para determinar longitud, perímetro o área de formas compuestas (MINEDU, 2016).

Carranza y Malaspina (2015) señalan que en Perú existen pocas investigaciones referidas a Didáctica de la Matemática. De igual forma, Flores y Gaita (2014), señalan que en este país existen evidencias de la presencia de un modelo conductista en los planes de formación de profesores del nivel primario y secundario.

Teniendo en cuenta lo reportado anteriormente y considerando que se necesita profundizar en la exploración del conocimiento profesional que posee el profesorado de secundaria en Perú, nos planteamos la siguiente pregunta de investigación: ¿Cuál es el conocimiento especializado que moviliza el profesorado de educación secundaria en formación cuando enseña polígonos? Para ello, nos planteamos como objetivo describir y analizar el conocimiento especializado que evidencia una profesora de matemática de educación secundaria en formación, cuando enseña polígonos, a través de un estudio de caso.

Por otro lado, entre las investigaciones relacionadas con el conocimiento del profesorado de matemática sobre contenidos de geometría y las concepciones que tiene respecto a la enseñanza y el aprendizaje de esta disciplina encontramos a Aguilar-González (2015), quien aborda la identificación y comprensión del conocimiento especializado que muestra una maestra de educación primaria sobre la clasificación de 
las figuras planas, usando el modelo MTSK para explorar las posibles relaciones entre sus subdominios y obtener una visión integrada del conocimiento especializado de la maestra. En dicho trabajo se detectan las concepciones sobre la enseñanza y aprendizaje de las matemáticas y su relación con los subdominios del conocimiento especializado de la maestra para la enseñanza de polígonos. Para este autor la identificación y exploración de las relaciones entre las concepciones sobre la enseñanza y el aprendizaje de las matemáticas y los elementos de conocimiento permiten comprender y explicar el conocimiento que la maestra moviliza en su salón de clases.

Escudero (2015) explora el conocimiento especializado que movilizan docentes de matemáticas de secundaria y señala que el conocimiento de los temas es el subdominio del que se obtiene más información, ya que ocupa el primer lugar de aparición respecto a los otros subdominios y se relaciona con todos los subdominios del MTSK. La autora menciona que este subdominio podría ser el eje sobre el cual se relacionan y organizan los demás conocimientos del MTSK. Asimismo, resalta la importancia de la presencia del conocimiento de la enseñanza de las matemáticas pues considera que conocer aspectos de enseñanza relacionados con el contenido matemático ayuda a reconocer aspectos inherentes a la práctica del profesorado de matemáticas, lo cual se vincula con el conocimiento de las características del aprendizaje en matemáticas, diferenciándose del conocimiento de pedagogía y didáctica general. Finalmente, señala que reconocer aspectos específicos de la matemática resultan útiles para el profesorado al plantear la enseñanza de contenidos matemáticos.
Lizarde, Hernández y Reyes (2019) indican que la formación de docentes para la enseñanza de las matemáticas en la educación primaria en México sigue siendo una tarea no resuelta, en tanto la clarificación sobre los componentes del conocimiento especializado del profesorado de matemáticas solo está enunciada teóricamente, pero su concreción en cuanto a la formación práctica requiere aún posteriores esfuerzos.

Por otro lado, Carreño y Climent (2010) señalan que el conocimiento geométrico del futuro profesorado de matemáticas, en general, es limitado conceptualmente y, por ello, carente de redes matemáticas complejas y de relaciones inclusivas entre varios objetos geométricos. Posteriormente, Carreño y Climent (2019) hacen una exploración del conocimiento especializado de futuro profesorado de secundaria sobre las definiciones de cuadriláteros, y muestran que sus definiciones tienden a ser descriptivas e incompletas.

En cuanto a las estrategias didácticas del profesorado peruano, Rivas y Luzardo (2017) señalan que la estrategia más usual es la exposición, coincidiendo en este punto con otros estudios como los de Gamboa y Ballestero (2010) o Pochulu y Font (2011). Estos autores también señalaron que la enseñanza de los polígonos en la escuela se sigue haciendo de manera memorística sin el uso de recursos eficaces para la enseñanza de la geometría.

\section{Marco teórico}

El conocimiento del profesorado ha sido explorado ampliamente en los últimos 35 años, desde los trabajos iniciales de Shulman (1986), que consideraba la necesidad de contemplar al personal docente como especialista con gran comprensión de 
la disciplina, y sus procesos de enseñanza y aprendizaje. Con base en una visión intrínseca de la especialización docente (Scheiner, Montes, Godino, Carrillo y Pino-Fan, 2019), que asume que el conocimiento del profesorado es especializado en tanto que le sea útil para su quehacer profesional, Carrillo et al. (2018) propusieron el modelo MTSK. Este modelo de análisis del conocimiento profesional propone, desde un paradigma interpretativo, una mirada hacia el conocimiento del profesorado desde tres dominios: conocimiento matemático $(\mathrm{MK}$, todos los acrónimos del modelo corresponden a sus siglas en inglés), conocimiento didáctico del contenido ( $\mathrm{PCK}$ ), y dominio afectivo. En este trabajo no profundizaremos en el último dominio que, si bien permea todo el conocimiento del profesorado, posee una naturaleza diferente de los dos anteriores y requiere de un tipo de aproximación diferente.

El dominio del MK contempla el conocimiento matemático puramente disciplinar que el profesorado usa en cualquier actividad ligada a su profesión. Así, obviamente, este conocimiento debe trascender con creces el conocimiento que sus estudiantes construirán, tanto en cantidad como en naturaleza, permitiendo al profesorado gestionar y guiar los procesos de aprendizaje y enseñanza de sus estudiantes. Este dominio se descompone en tres subdominios: conocimiento de los temas (KoT), conocimiento de la estructura matemática (KSM) y conocimiento de la práctica matemática (KPM).

El subdominio KoT abarca un conocimiento local al contenido que se enseña, en el sentido de conocer los conceptos, definiciones, fenómenos, procedimientos y registros de representación relacionados con el tema. También contempla el conocimiento de propiedades matemáticas $\mathrm{y}$ sus fundamentos, no solo como objeto de aprendizaje, sino como aquello que permite al profesorado reflexionar y construir nuevos conocimientos matemáticos.

El KSM aporta un conocimiento de índole más global, que abarca el conocimiento que el profesorado posee sobre las conexiones entre elementos matemáticos. Estas conexiones pueden ser o, entre elementos del mismo tema matemático, en términos de aumento o disminución de la complejidad, o entre elementos de temas diferentes. Así, se consideran cuatro tipos de conexiones: las de simplificación, las de complejización, las auxiliares y las transversales. Las dos primeras hacen referencia al establecimiento de relaciones con contenidos curricularmente previos o posteriores, de modo que sean de utilidad para la enseñanza del contenido que se enseña en un momento dado. Las conexiones auxiliares vienen dadas por la introducción de un concepto, técnica, definición o propiedad, con una función meramente coadyuvante al elemento matemático que es el centro de la reflexión en un momento dado de una clase. Por último, las conexiones transversales vienen dadas por la relación entre el contenido objeto de enseñanza y aprendizaje con elementos matemáticos que aparecen de forma transversal (explícita o implícitamente) en la matemática escolar.

El KPM abarca el conocimiento matemático de índole sintáctica, es decir, ligado a las reglas de construcción de un nuevo conocimiento matemático. Por ejemplo, abarca el conocimiento de diferentes formas de validación, como la demostración, aspectos relacionados con la heurística en resolución de problemas o prácticas matemáticas centradas en la construcción de teoría.

El dominio del PCK contempla conocer la matemática desde la perspectiva 
centrada en su enseñanza y aprendizaje. Este dominio se divide en tres subdominios: el conocimiento de la enseñanza de las matemáticas (KMT), el conocimiento de las características del aprendizaje matemático (KFLM) y el conocimiento de los estándares de aprendizaje matemático (KMLS).

El KMT se centra en las diversas técnicas, recursos y teorías que el profesorado puede conocer para gestionar la enseñanza del contenido. Cabe destacar que, en este subdominio, al igual que en todo el modelo, quedan excluidos los elementos no ligados al contenido matemático, debido a la perspectiva intrínseca de la especialización adoptada (Carrillo et al., 2018; Scheiner et al., 2019). Este subdominio contempla las especificidades del conocimiento del profesorado sobre el uso de GeoGebra o regletas, así como su conocimiento de diversos elementos de teorías de enseñanza de la matemática.

El KFLM contempla el conocimiento del profesorado acerca de cómo se aprende el contenido. Así, este subdominio abarca tanto conocimientos de teorías personales e institucionalizadas sobre cómo transcurre el aprendizaje de estudiantes en general o de alumnado concreto, como conocimiento de la dimensión emocional del aprendizaje, así como las formas de interacción estudiantiles con diversos contenidos matemáticos.

El KMLS refleja el conocimiento que el profesorado posee de las orientaciones dadas por autoridades de diversos niveles acerca de qué debe aprender el alumnado en cierto momento. Esto incluye tanto el conocimiento curricular propuesto por Shulman (1986), como el conocimiento de estándares de aprendizaje propuestos por asociaciones de docentes (e.g. NCTM), así como el conocimiento de las concreciones del currículo impuestas o propuestas desde el centro educativo en el que se imparte clase, si las hubiera.

\section{Metodología}

Este trabajo se ha desarrollado desde una perspectiva cualitativa, asumiendo un paradigma interpretativo, ya que el propósito de nuestra investigación es explorar, describir y explicar (McMillan y Schumacher, 2007), para así comprender al profesorado. El foco de esta investigación es analizar el conocimiento de la maestra a través de un proceso de interpretación en el que la sensibilidad teórica (Strauss y Corbin, 1994) será crucial para dar significado a los datos recogidos en cada uno de los episodios. El diseño de la investigación es a través de un estudio de caso de tipo instrumental (Stake, 1995). Esta clase de estudio permite profundizar en la comprensión de un tema determinado, en este caso en la comprensión del conocimiento especializado del profesorado acerca de polígonos.

El caso elegido fue una futura profesora de matemática del nivel secundario, que cursaba el décimo ciclo de la carrera de educación secundaria con especialidad en matemática y física, y se encontraba realizando su práctica preprofesional, la que le permite ejercer el rol de docente en una institución educativa. Esta profesora fue elegida de manera intencionada por haber sido una estudiante destacada en su promoción y por haber aceptado colaborar con nuestra investigación de manera voluntaria.

En este trabajo, para la recogida de información optamos por realizar una observación no participante, registrada a través de videograbaciones de sesiones de clase, las cuales fueron posteriormente transcritas y constituyeron la principal fuente de información del estudio. Se observaron 3 
sesiones de clase relativas a los polígonos en el curso de Práctica preprofesional que forma parte del plan de estudios de Educación Secundaria en la especialidad de Matemática y Física del Instituto Pedagógico Nacional de Monterrico, en el año 2019.

A continuación, mostramos una breve descripción del contenido de las sesiones, precisando los elementos conceptuales trabajados en cada una de ellas.

Para el análisis de los datos de nuestro caso utilizamos los dominios, subdominios y categorías descritas en Carrillo et al. (2018), adaptándolas al contenido de polígonos.

El tratamiento y análisis de los datos se desarrolló siguiendo la siguiente cronología: en primer lugar, se videograbaron y transcribieron las sesiones de clase, lo que permitió una primera sensibilización de los investigadores con los datos; en un segundo momento, se identificaron los episodios más ricos en información, de manera que, además de profundizar en la sensibilización con los datos, supuso su organización; en un tercer momento, se hizo un análisis de contenido (Bardín, 1998) por cada integrante del equipo de investigación de forma independiente. Finalmente, se realizó un proceso de triangulación por personal experto (Flick, 2007), por parte de sujetos investigadores externos expertos familiarizados con el modelo MTSK y con los distintos elementos de conocimiento profesional relativos a los polígonos.

\section{Análisis y resultados}

En esta parte mostramos el análisis de 3 episodios que seleccionamos de las sesiones de clase, por contener información relevante para nuestro estudio. Para el análisis usamos el modelo MTSK, el cual nos permitió sistematizar la información obtenida y nos ayudó a comprender cómo está organizado el conocimiento que moviliza nuestra profesora participante cuando enseña polígonos.

\section{Síntesis del episodio 1}

La profesora explora los conocimientos previos de sus estudiantes acerca de los

Tabla 1

Descripción de las clases

\begin{tabular}{ll}
\hline Sesiones & Descripción de la sesión \\
\hline Sesión 1 & $\begin{array}{l}\text { La docente exploró los conocimientos previos de sus estudiantes acerca de los polígonos a } \\
\text { través de una lluvia de ideas. Luego, mostró distintas definiciones de polígonos, así como } \\
\text { los principales elementos y características de dichas figuras geométricas. }\end{array}$ \\
\hline Sesión 2 & $\begin{array}{l}\text { La docente abordó dos tipos de clasificación de los polígonos, según su número de lados y } \\
\text { según sus características en regular e irregular. También, presentó dos propiedades de los } \\
\text { polígonos convexos, sumas de ángulos interiores y suma de ángulos exteriores. }\end{array}$ \\
\hline Sesión 3 & $\begin{array}{l}\text { La docente abordó la propiedad de los polígonos convexos relacionada con su número de } \\
\text { diagonales. Luego, retomó la clasificación de los polígonos según sus características en } \\
\text { regular e irregular. Asimismo, abordó las propiedades de suma de ángulos interiores y exte- } \\
\text { riores en diferentes tipos de triángulos. }\end{array}$ \\
\hline
\end{tabular}

Nota: Fuente propia de la investigación. 
polígonos a través de una lluvia de ideas, a fin de rescatar los conocimientos que traen. Luego, muestra distintas definiciones de polígonos, así como sus principales elementos y características.

\section{Análisis del episodio 1}

La profesora (P) indaga acerca del contenido matemático a tratar en la sesión a través de la siguiente pregunta que plantea a sus estudiantes (A, no se distinguirá entre estudiantes) ¿qué es un polígono? Muestra el uso de la lluvia de ideas como una estrategia para rescatar los conocimientos previos sobre la definición de polígono, evidenciando conocimiento de la enseñanza de las matemáticas (KMT).

$P$ : ¿Qué es un polígono?

A: Es una figura plana que tiene lados.

(La profesora da oportunidad a varios estudiantes a dar su respuesta)

A: Es una figura geométrica de primer plano.

A: Es un conjunto de segmentos unidos para formar una figura.

A: Es cualquier figura plana cerrada.

La docente, posteriormente, pregunta: ¿Cuál es la mínima figura que se puede formar?, aquí consideramos que la docente hace un uso informal del vocabulario matemático, ya que hace referencia a los límites de la definición de polígono, invitando a explorar qué figuras no serían polígonos, mostrando KoT relativo a definiciones. Posteriormente evidencia KoT sobre las propiedades de los polígonos, en concreto, sobre el mínimo número de lados que pueden tener:
P: ¿Podrías formar una figura con dos lados?

A: No podría formar, sería rectas paralelas, un ángulo.

P: Para formar un polígono lo mínimo es tres lados.

Posteriormente, la docente presenta a sus estudiantes dos definiciones que, si bien no son precisas, pretenden reforzar la idea del mínimo número de lados en un polígono:

P: Los polígonos según su referencia son figuras planas, son puntos o vértices formando una figura, mínimo podría ser un triángulo, un cuadrado, un rectángulo.

$P:$ [...] es la figura formada por la reunión de segmentos de una recta determinada al unir 3 o más puntos"

En estas definiciones, además de movilizar conocimiento de los temas sobre los límites de la definición de polígono, también moviliza elementos que permiten definir lados y vértices, que posteriormente podrían permitir definir al polígono con mayor formalismo.

En un segundo momento de la sesión, la docente muestra varios polígonos en cartulina como se muestra la Figura $1 \mathrm{y}$ empieza a preguntar a sus estudiantes sobre los elementos que tiene un polígono. Al ser ella quien ha elegido los polígonos, muestra conocimiento de los temas ligado a los registros de representación gráficos. Asimismo, manifiesta conocimiento de la enseñanza de las matemáticas (KMT), dado que se hace uso de una tarea diseñada por ella misma, sobre la que pretende discutir los elementos que componen los polígonos, poniendo cierto énfasis en la forma de notar algunos de ellos, iniciando con una pregunta 
abierta que fomenta respuestas de muy diversa índole:

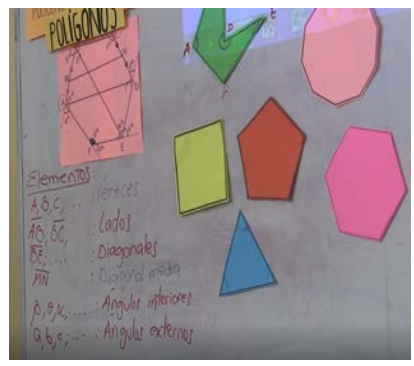

Figura 1. Polígonos.

Nota: Fuente propia de la investigación.

$P: ¿ Q u e ́$ elementos tienen los polígonos?

A: Grados

$P$ : ¿Cómo?

$P:$ ¿El polígono tiene grados?

A: No, lados, vértices, ángulos, diagonales, aristas

P: ¿Aristas?

P: Aristas es una figura tridimensional acuérdate

$P:$ Acuérdate que a los vértices se les denomina con letras mayúsculas

En el siguiente extracto de información, la docente evidencia conocimiento de los temas (KoT) cuando menciona los elementos de las figuras planas, aunque usa notación matemática informal, llamando segmentos a los lados.

P: Ahi tenemos dos tipos de diagonales.

$P:$ ¿Cuál es la primera?

$P: A B, B C$ son los lados o segmentos.

$P:$ ¿Qué sería BE?

$P$ : Aquí tengo el punto $B$ y lo uno con el punto opuesto a él y sería la diagonal

$P$ : ¿Qué más se puede observar?

A. cuerda....

$P$ : ¿Qué segmento es MN? ¿Qué será MN en la imagen? Te das cuenta de este punto
$N$. ¿Qué sucede con el punto $N$ en el sector $C D$ ?

P: Perfecto. Es el punto medio, por ende, $M$ también es el punto medio

$P:$ ¿Cómo se va a llamar esta línea?

P: Diagonal media. El segmento MN es la diagonal media

Vemos cómo la profesora afirma que existen dos tipos de diagonales: la diagonal de un polígono en el sentido habitual y otra "diagonal media", que parece corresponder a la mediana, asume que sus estudiantes identifican dichas diagonales, pues no se observa que exista aclaración posterior del tema. Esto supone un indicio para indagar con la profesora, dado que la terminología es poco habitual, pudiendo existir un conocimiento de los temas ligado a la nomenclatura de los elementos. Asimismo, la introducción de la mediana en este momento de la formación puede informar del KMLS.

\section{Síntesis del episodio 2}

La profesora aborda dos tipos de clasificación de los polígonos, según su número de lados y según sus características en regular e irregular, usando figuras de polígonos en cartulina o trazadas en la pizarra. Además, presenta dos propiedades de los polígonos convexos, sumas de ángulos interiores y suma de ángulos exteriores. La profesora usa figuras que coloca o traza en la pizarra y realiza constantemente preguntas a sus estudiantes.

\section{Análisis del episodio 2}

La profesora evidencia un KoT relativo a las propiedades de los polígonos al mostrar al alumnado la clasificación de los polígonos según su número de lados. 
Además, evidencia un KMT respecto al uso de una estrategia de enseñanza al presentar a sus estudiantes algunos polígonos dibujados en cartulina (pentágono, hexágono, octógono y decágono) y pedir que indiquen los nombres de dichos polígonos a partir del número de lados que tienen. Observamos que la docente no hace ninguna aclaración cuando muestra como ejemplo un octógono regular y uno de sus alumnos identifica el nombre de dicho polígono sin mencionar que es regular, como se observa en el siguiente extracto de información:

P: ¿Cómo se llama la figura de 8 lados iguales?

A: Octógono

En otro momento de la clase, la docente evidencia un KoT respecto a las propiedades de los polígonos, al abordar la clasificación de los polígonos en regulares e irregulares haciendo preguntas a sus estudiantes y enfatizando que justifiquen las características que presentan los polígonos regulares. Asimismo, evidencia un KMT respeto al uso de una estrategia de enseñanza, al hacer preguntas constantes al alumnado para tratar de asegurar el conocimiento sobre los polígonos regulares, lo cual también podría dar un indicio de un KFLM al tener en cuenta cómo sus estudiantes interactúan con el contenido matemático, tal como se muestra en el siguiente extracto de información:

P: ¿Este polígono es regular o irregular? (La profesora muestra figuras en la pizarra) A: Irregular

$P:$ ¿Por qué son irregulares?

A: Porque no todos sus lados son iguales

P: Muy bien. Porque todos sus lados no tienen la misma medida.
Por otro lado, la profesora evidencia un KMT relativo al uso de una estrategia de enseñanza al pedir que sus estudiantes comenten sobre lugares o cosas del entorno que tienen forma de polígonos y describan las características de dichas figuras; así como un KMLS al tomar en cuenta la propuesta curricular de la educación básica, donde se indica que todo contenido a enseñar se debe relacionar con situaciones de nuestro entorno, tal como se muestra en el siguiente extracto de información:

$P: ¿$ ¿stedes han visto cualquiera de estas figuras $u$ otras en la vida diaria o cotidiana? A: Si

$P:$ ¿Dónde?

A: Los octógonos

P: En los octógonos. Muy bien. ¿Está de moda cierto? ¿Por qué? Cuéntanos.

A: Porque está en los empaques de comida P: Muy bien. A ver, entonces vemos las figuras geométricas a nuestro alrededor. Por ejemplo, en el salón vemos varias figuras, varios polígonos, ¿cierto? La mayoría son de 4 lados, algunos de 3 lados.

La docente evidencia un KoT respecto a las propiedades de polígonos convexos como suma de ángulos interiores en un polígono regular y medida de un ángulo interior, a través de preguntas que hace a los estudiantes, por ejemplo ¿recuerdan cómo se cumplen estas propiedades en triángulos equiláteros y cuadrados? Al hacer constantemente preguntas a sus estudiantes, la docente evidencia un KMT relativo al uso de una estrategia de enseñanza, como se muestra en el siguiente fragmento:

P: ¿Te acuerdas cómo hallamos la medida de la suma de los ángulos internos de un polígono? 
A: 180 por $n$ menos ...

P: [...] en el triángulo que es el polígono más pequeño, ¿cuánto miden sus ángulos internos? ¿la suma?

A: $180^{\circ}$ (La profesora marca los 3 ángulos en un triángulo de cartulina que está pegado en la pizarra)

P: Como lo dijo su compañero, un polígono regular de 3 lados es un triángulo equilátero. ¿Verdad? Por ende, ¿cuánto vale su ángulo interior de un triángulo equilátero? A: $60^{\circ}$

P: $60^{\circ}$ y como son iguales $60^{\circ}$ por $3 \ldots$

A: $180^{\circ}$

P: $180^{\circ}$, muy bien

En otro momento de la clase, la profesora trata de que sus estudiantes conozcan una forma práctica para determinar la suma de ángulos interiores en un pentágono y les guía para que dividan al pentágono en triángulos que no se intersecan entre sí, evidenciando así un KMT relativo a otra estrategia de enseñanza. En esta parte, la profesora observa que sus estudiantes tienen dificultades y recurre a una figura para aclarar la confusión al momento de dividir el pentágono, evidenciando así un KFLM al tomar en cuenta las dificultades que presentan, tal como se muestra en el siguiente fragmento:

P: En el caso del pentágono, ¿cuánto miden sus ángulos interiores?

A: Si, yo lo dividí. Podemos dividirlo para formar triángulos y como los ángulos internos de un triángulo suman $180^{\circ}$...

P: Muy bien y de alli lo suman. Una estrategia que utiliza su compañera para saber cuánto vale el ángulo interno de un pentágono es formar triángulos.

P: Muy bien. A ver, ¿cómo hallamos en el caso de un pentágono?
A: Podríamos multiplicar $180^{\circ}$ por 5 porque hay 5 triángulos

P: ¿180 por 5? ¿Aquí hay 5 triángulos?

A: Hay 3

P: Si lo unes así, me salen 3 triángulos. (La profesora indica la división en el pentágono pegado en la pizarra)

A: $540^{\circ}$ ¿Es $540^{\circ}$ grados?

P: 540 grados. A ver como lo hiciste ...

A: Dividí en un triángulo y un cuadrilátero.

$Y$ como el triángulo vale $180^{\circ}$ y el cuadrilátero vale $360^{\circ}$, lo sume ambos y me dio $540^{\circ}$ P: Muy bien. (La profesora indica el triángulo $A B C$ y el cuadrilátero $A C D E$ en la figura pegada en la pizarra)

P: Ok. Ahora ¿hay una fórmula general para hallar la suma de los ángulos internos de un triángulo, cuadrilátero o hexágono? ¿Cuál sería la fórmula?

A: $180^{\circ}$ por $n$ menos 2

$P$ : ¿Qué es $n$ ?

A: El número de lados

En otro momento, la profesora propone comprobar la fórmula mencionada anteriormente para el triángulo, pero el estudiantado pide que se compruebe para el dodecágono y el icoságono. Uno de los estudiantes da su respuesta, pero menciona que ha usado su calculadora. Aquí podemos decir que la profesora evidencia un KMT, pues usa el método inductivo como estrategia para ayudar a sus estudiantes a recordar la fórmula de la suma de los ángulos interiores de un triángulo y luego hace que usen la fórmula a través de un ejemplo. De manera similar, señala a que es igual la suma de los ángulos externos de un polígono y pide que comprueben este resultado en algunos polígonos conocidos, por ejemplo, triángulos, cuadriláteros, pentágonos; pero a partir de la relación que existe entre un ángulo interior y un ángulo exterior. Cabe resaltar que todos 
los ejemplos que propone la docente son con polígonos regulares, lo cual puede ocasionar una generalización incorrecta en estudiantes que asuman que estas propiedades se cumplen en polígonos que no son regulares, tal como se muestra en el siguiente fragmento:

P: Si su compañero me dijo que la suma era $540^{\circ}$, ¿cuánto vale uno de sus ángulos internos? ¿uno cuánto sería? ¿un ángulo interno? ¿cuánto sumaba todo?

A: $540^{\circ}$

P: Si son 5 ángulos y deben ser iguales, ¿qué tengo que hacer?

A: Dividir

P: Muy bien. Divide. ¿Cuánto saldría?

A: $108^{\circ}$

P: $108^{\circ}$. Miss entonces, ¿el ángulo interno sería $108^{\circ}$ ? ¿el ángulo externo cuánto valdría allí? El ángulo externo recuerda que tiene que sumar $180^{\circ}$

A: $72^{\circ}$

P: $72^{\circ} \cdot ¿ 72^{\circ}$ por 5 cuánto sería?

A: $360^{\circ}$

P: Muy bien. También suma $360^{\circ}$, ¿cierto? Muy bien.

Finalmente, la profesora da indicios de un KFLM, pues trata de asegurar el aprendizaje de sus estudiantes repitiendo varias veces lo mismo y dando varios ejemplos. También podríamos decir que la profesora evidencia un KMT referido al uso de una estrategia de enseñanza al hacer una síntesis de lo trabajado en la sesión proponiendo a sus estudiantes completar una ficha que elaboró previamente, como se muestra en el siguiente fragmento:

P: Vamos a hacer algo a continuación. En la fichita que les entregué están allí las propiedades que se cumplen en los polígonos, ¿cuál es la primera?
A: La suma de ángulos internos

$P$ : ¿Cuánto es? ¿cuál es la formulita?

A: 180 grados por $n$ menos 2

P: Saben ... ¿cuánto es la suma de los ángulos externos siempre?

A: $360^{\circ}$

\section{Síntesis del episodio 3}

La profesora aborda la propiedad de los polígonos convexos relacionada con su número de diagonales. Luego, retoma la clasificación de los polígonos según sus características en regular e irregular. Asimismo, aborda las propiedades de suma de ángulos interiores y exteriores en diferentes tipos de triángulos.

\section{Análisis del episodio 3}

La docente muestra la figura de un triángulo en la pizarra e introduce el término diagonal a través de las siguientes preguntas: ¿El triángulo tiene diagonales? ¿Se pueden trazar diagonales en el triángulo? La docente explica qué es una diagonal de un polígono, evidenciando un KoT relativo a las características de las diagonales de los polígonos, destacando la condición que cumplen los puntos extremos de una diagonal. Asimismo, la docente fomenta la participación del alumnado a través de preguntas, manifestando un KMT relativo al uso de estrategias de enseñanza, tal como se muestra en el siguiente fragmento:

$P:$ ¿El triángulo tiene diagonales? ¿Se pueden trazar diagonales en el triángulo?

A: No se puede

$P$ : ¿Por qué no se puede?

A: Para trazar diagonales debe ser desde un punto a un vértice que no sea el que esté al costado, pero en el triángulo en los tres lados está al costado. 
P: Muy bien, son consecutivos. Sí, aquí, por ejemplo, no hay vértice opuesto, el vértice que está continúo, aquí tiene dos, adyacentes se puede decir, no se puede formar diagonales.

La docente enfatiza las características que debe cumplir un polígono para ser regular, evidenciando un KMT al usar como estrategia de enseñanza el hacer preguntas que ayuden al alumnado a comprender el contenido tratado.

P: Y para que sea un polígono regular ¿cómo deben ser sus ángulos?

A: Iguales

$P:$ ¿Y sus lados?

A: Iguales

Luego, la docente menciona que un polígono regular debe ser equiángulo $\mathrm{y}$ equilátero, haciendo énfasis a las condiciones de lados y ángulos iguales, mostrando un KoT relativo a las definiciones y características de polígonos regulares. También, intenta ser precisa con los términos usados por sus estudiantes, utiliza un vocabulario coherente adecuado asociado a los tipos de polígonos, mostrando así un KoT relativo al uso adecuado del vocabulario matemático, como se evidencia en el siguiente extracto de información:

P: Equiángulo y equilátero, entonces sería un triángulo ¿de qué tipo?

A: regular

P: Un polígono regular, muy bien

Mas adelante, la docente evidencia un KoT relativo a la clasificación de los triángulos según las medidas de sus ángulos interiores, al precisar que los triángulos pueden ser: oblicuángulos (acutángulos y obtusángulos) o rectángulos. Asimismo, muestra un KMT al usar como estrategia de enseñanza el uso de preguntas de manera constante, proporcionando al alumnado la oportunidad de reflexionar sobre sus argumentos cuando participan. La docente también evidencia un KFLM referido al conocimiento de las fortalezas y dificultades de estudiantes, al indagar si tienen dificultades para analizar e identificar la clasificación de los triángulos que se está tratando, tal como se muestra en el siguiente fragmento:

P: Claro, según sus ángulos ¿Cómo se clasifican los triángulos?

A: Hay dos: Oblicuángulos y rectángulos; $y$ dentro de los oblicuángulos hay dos tipos. $P$ : Dentro de los triángulos oblicuángulos hay dos tipos de triángulos ¿cuáles son?

A: Acutángulo y obtusángulos

$P: ~ ¿ C u a ́ n d o$ es un triángulo es acutángulo? A: Cuando todos los ángulos internos son menores que 90 grados.

$P$ : ¿Son menores a 90 grados?

A: Todos los ángulos internos son menores a 90 grados

$P: ~ ¿ C u a ́ l$ es el triángulo obtusángulo?

A: Cuando sus ángulos son menores, ¿cierto?

A: Cuando son mayores que noventa y menores a 180 grado

P: Cuando uno de ellos es mayor que noventa y menor a 180 grados, porque no todos pueden ser mayores que a 180 grados. Muy bien. ¿A qué se le llama triángulo rectángulo?

A: Cuando uno de sus ángulos mide 90 grados

En el siguiente diálogo, la maestra muestra un conocimiento de la suma de los ángulos interiores de un triángulo, evidenciado un KoT relativo a propiedades de los 
ángulos en un polígono convexo. Además, muestra un KPM referido al conocimiento de una forma de demostración, al conocer y realizar la demostración visual de la suma de ángulos interiores en un triángulo con sus estudiantes, tal como se muestra en el siguiente fragmento:

P: ¿Por qué tú me diste esa relación? ¿A qué se debe?

A: Porque la suma de todos sus ángulos es $180^{\circ}$.

P: Haber vamos a comprobarlo. Aquí tenemos un triángulo. (La profesora coloca alfa, beta y theta con letras griegas en los ángulos internos) ¿Cuáles son sus ángulos? A: Beta, alfa y theta.

P: Miramos. Si retiro los ángulos alfa, beta $y$ theta del triángulo, ¿qué sucede si yo junto a los tres ángulos nuevamente? (La profesora retira las tres partes que corresponden a los ángulos del triángulo y los coloca uno a continuación de otro)

A: Se forma un ángulo de $180^{\circ}$.

P: Perfecto formamos un ángulo de 180 grados. ¿Qué estamos demostrando?

A: Que todos los ángulos internos de un triángulo siempre van a valer 180 grados P: Perfecto. La suma de los ángulos internos en este caso ... ¿theta más alfa más beta cuánto suman?

\section{Conclusiones}

En este estudio nos hemos preguntado por los conocimientos que el profesorado de matemáticas en formación inicial pone en juego al enseñar polígonos en un aula de educación secundaria. Del análisis realizado podemos concluir que una profesora de matemática de educación secundaria, en formación inicial, al enseñar polígonos evidencia el uso de diferentes dominios y subdominios del MTSK, a excepción del subdominio KSM referido al uso de conexiones entre los conocimientos matemáticos previos y posteriores; cuya identificación creemos requiere de preguntas específicas que habría que realizar a la docente.

El MTSK nos permitió profundizar en la comprensión del conocimiento que moviliza el profesorado de matemáticas en formación inicial cuando enseña polígonos, así como concretar un análisis a través de la categorización y descripción de los subdominios de dicho modelo. Consideramos que este análisis contribuirá a la identificación y comprensión del conocimiento especializado del profesorado de matemáticas de educación secundaria en formación inicial, los cuales deben ser tomados en cuenta en su formación profesional. Asimismo, consideramos que ayudará a que el personal docente en formación inicial reflexione sobre la necesidad de tener un conocimiento matemático y didáctico sobre el contenido a enseñar.

A partir del análisis realizado, teniendo en cuenta que nuestra pregunta de investigación gira en torno a comprender el conocimiento especializado que moviliza una profesora en formación cuando enseña polígonos en educación secundaria, podemos resaltar, en términos generales, la importancia que tiene el KoT como base para la enseñanza desde una perspectiva matemática $(\mathrm{MK})$ y didáctica (PCK). En este punto, coincidimos con Escudero (2015), quien señala haber encontrado en su estudio que la mayoría de los indicadores estaban relacionados con el KoT, y se detectaron múltiples relaciones entre el KoT y los otros subdominios del MTSK.

En nuestro trabajo, la profesora de matemática en formación evidencia tener conocimiento de conceptos claves para 
definir los polígonos, clasificar polígonos según su número de lados y según sus características en regulares e irregulares; así como para describir las propiedades relacionadas con los polígonos convexos como la suma de ángulos interiores y de ángulos exteriores y el número de diagonales. Además, la docente participante usa representaciones verbales y simbólicas al presentar a los polígonos, indicando sus principales elementos: lados, ángulos, vértices y diagonales. Aquí también coincidimos con Escudero (2015), quien menciona que el conocimiento y uso de diferentes registros de representación permite reconocer aspectos específicos de la matemática que resultan útiles al profesorado en formación para plantear diversos tratamientos didácticos al enseñar un tema matemático.

Asimismo, consideramos, en la línea de Escudero (2015), que el conocimiento de los temas puede estar ligado directamente al dominio del conocimiento didáctico del contenido, y en particular al conocimiento de la enseñanza de la matemática y de las características del aprendizaje de las matemáticas. En nuestro trabajo, el segundo subdominio que apareció reiteradamente fue el conocimiento de la enseñanza de las matemáticas, aportando información importante para conocer la enseñanza de la maestra participante. Algunos conocimientos que destacan son el uso de estrategias de enseñanza como generar una lluvia de ideas; promover la participación constante del alumnado y usar material concreto para visualizar los polígonos, identificar sus principales elementos y aclarar dudas y dificultades que presentan sus estudiantes. Otra estrategia fue hacer preguntas orientadoras, por ejemplo, preguntó por la relación que encuentran entre los elementos de los polígonos, para luego abordar sus propiedades; también, hizo preguntas para aclarar la clasificación de los polígonos según sus características, para diferenciar polígonos regulares e irregulares, así como para generalizar la propiedad de suma de ángulos interiores en polígonos convexos. El uso de estas estrategias de enseñanza por parte de la docente en formación da indicios de un conocimiento de las características del aprendizaje de las matemáticas, ya que está tomando en cuenta las dificultades que suele tener el estudiantado cuando interactúa con contenidos matemáticos. Además, promovió un diálogo para ayudar a concretar el conocimiento que se está tratando. Por el análisis de los resultados, consideramos que los subdominios KMT y KFLM mantienen una relación estrecha que se sostiene en un vínculo directo con el KoT.

Finalmente, consideramos que este estudio aporta a la formación inicial de profesorado de matemática de educación secundaria, ya que permite comprender que los contenidos matemáticos a enseñar deben ser tratados desde los dominios matemático y didáctico. Por ende, en la formación del profesorado se debe fortalecer el diseño de tareas y estrategias de enseñanza que desarrollen habilidades vinculadas al conocimiento de ambos dominios. Asimismo, este trabajo aporta a nuevas investigaciones que permitan comprender mejor el conocimiento del personal docente en formación inicial desde el modelo MTSK, así como el diseño de nuevas propuestas que le ofrezcan una formación profesional sólida al futuro profesorado de matemática de educación secundaria. 


\section{Financiamiento}

Universidad de Lima, Instituto de Investigación Científica, Perú. PI:51.001.2019

\section{Agradecimiento}

Agradecimiento al Centro de Investigación COIDESO de la Universidad de Huelva y al Ministerio de Ciencia, Innovación y Universidades, del Gobierno de España (Proyecto: RTI2018-096547-B-I00).

\section{Consentimiento informado}

La docente participante fue informada acerca de la investigación, aceptó participar y firmó un consentimiento voluntario.

\section{Conflicto de intereses}

Los autores declaran no tener algún conflicto de interés.

\section{Declaración de la contribución de los autores}

Todos los autores afirmamos que se leyó y aprobó la versión final. El porcentaje total de contribución para la conceptualización, preparación y corrección de este artículo fue el siguiente: E.A.C. $20 \%$, M.B.S. $20 \%$, J.C.L.R. $20 \%$, I.T.C. $20 \%$ y M.M. $20 \%$.

\section{Declaración de disponibilidad de los datos}

Los datos que respaldan los resultados de este estudio serán puestos a disposición por el autor correspondiente [E.A.C.], previa solicitud razonable.

\section{Referencias}

Aguilar-González, A. (2015). El conocimiento especializado de una maestra sobre la clasificación de las figuras planas. Un estudio de caso [Tesis Doctoral]. Universidad de Huelva, Huelva, España.

Ball, D. L., Thames, M. H., \& Phelps, G. (2008). Content knowledge for teaching: What makes it special? Journal of Teacher Education, 59(5), 389-407. https://doi. org/10.1177/0022487108324554

Bardin, L. (1998). L'analyse de contenu. Presses Universitaires de France - Le Psychologue, Paris.

Carranza, C., \& Malaspina, U. (2015). Perú: A Look at the History of Mathematics and Mathematics Education. In H. Rosario, P. Scott y B. Vogeli (Eds.), Mathematics and its Teaching in the Southern Americas (pp. 363380). World Scientific Pub. Co. https://doi. org/10.1142/9789814590570_0015

Carrillo-Yañez, J., Climent, N., Montes, M., Contreras, L., Flores-Medrano, E., Escudero-Ávila, D., Vasco, D., Rojas, N., Flores, P., Aguilar-González, A., Ribeiro, M., \& Muñoz-Catalán, M. (2018). The mathematics teacher's specialised knowledge (MTSK) model. Research in Mathemtics Education, 20(3), 236253. doi:10.1080/14794802.2018.1479981

Carreño, E., \& Climent, N. (2010). Conocimiento del contenido sobre polígonos de estudiantes para profesor de matemáticas. PNA, 5(1), 183-195.

Carreño, E., \& Climent, N. (2019). Conocimiento especializado de futuros profesores de matemáticas de secundaria. Un estudio en torno a definiciones de cuadriláteros. PNA 14(1), 23-53.

Díaz, H. (2015). Formación docente en el Perú: Realidades y tendencias. Departamento de Marketing de Santillana.

Escudero, D. (2015). Una caracterización del conocimiento didáctico del contenido como parte del conocimiento especializado del profesor de matemáticas de secundaria [Tesis doctoral]. Universidad de Huelva, Huelva, España.

Flick, U. (2007). Introducción a la investigación cualitativa. Morata.

Flores, J. V., \& Gaita, C. (2014). Situación actual de la educación matemática en el Perú. Revista de matemática, ensino e cultura, 9(15), 82-95. http://www.rematec.net.br/index.php/ inicio/issue/current 
Gamboa, A., R., \& Ballestero, A. E. (2010). The Students' Perspective of Geometry Teaching and Learning in High School. Revista Electrónica Educare, 14(2), 125-142. https://doi. org/10.15359/ree.14-2.9

Lizarde, E., Hernández, J., \& Reyes, M. (2019). Ruta crítica en la construcción del MTSK. Meta-análisis del análisis didáctico de los docentes en formación inicial. En $X V$ Conferencia Interamericana de Educación Matemática (pp. 1-7). Universidad de Medellín. https:// conferencia.ciaem-redumate.org/index.php/ xvciaem/xv/paper/viewFile/275/10

Ministerio de Educación del Perú. (2016). Currículo Nacional de la Educación Básica. Lima, Perú. http://www.minedu.gob.pe/curriculo/pdf/curriculo-nacional-de-la-educacion-basica.pdf

Ministerio de Educación del Perú. (2010). Diseño Curricular Básico Nacional para la Carrera Profesional de Profesor de Educación Secundaria en la especialidad de Matemática. Lima, Perú: Dirección de Educación Superior Pedagógica. Área de Formación Inicial Docente.

McMillan, J., \& Schumacher, S. (2007). Investigación educativa (5. ${ }^{\mathrm{a}}$ ed.). Pearson, Addison Wesley.

Osorio, A. (2017). Perú: La formación inicial y continua de los profesores de matemáticas. Capacity and Networking Project 2016 International Commission on Mathematical Instruction. Cuadernos de Investigación y Formación en Educación Matemática, 12(16), 49-82. https://revistas. ucr.ac.cr/index.php/cifem/article/view/27543
Pochulu M., \& Font, V. (2011). Análisis del funcionamiento de una clase de matemáticas no significativa. Revista Latinoamericana de Investigación en Matemática Educativa, 14(3), 361-394. http://www.scielo.org.mx/pdf/reli$\mathrm{me} / \mathrm{v} 14 \mathrm{n} 3 / \mathrm{v} 14 \mathrm{n} 3 \mathrm{a} 5 . \mathrm{pdf}$

Rivas, L., \& Luzardo, H. (2017). Estrategias de enseñanza-aprendizaje y recursos tecnológicos utilizados en polígonos y geometría, en educación media general. Revista Aprendizaje Digital, 2(1), 47-57.

Scheiner, T., Montes, M. A., Godino, J. D., Carrillo, J., \& Pino-Fan, L. R. (2019). What makes mathematics teacher knowledge specialized? Offering alternative views. International Journal of Science and Mathematics Education, 37, 270. doi: 10.1007/ s10763-017-9859-6

Stake, R. (1995). The Art of case study. SAGE.

Shulman, L. S. (1986). Those who understand: Knowledge growth in teaching. Educational Researcher, 15(2), 4-14. 10.3102/0013189X015002004

Strauss, A., \& Corbin, J. (1994). Grounded Theory Methodology: An Overview. In N. K. Denzin, \& Y. S. Lincoln (Eds.), Handbook of Qualitative Research (pp. 273-285). SAGE.

\section{(@) $(\Theta \Theta$}

Conocimiento especializado del profesorado de matemática en formación inicial acerca de los polígonos (Elizabeth Advíncula-Clemente • Marisel Beteta-Salas • José León-Ríos • Isabel Torres-Céspedes $\bullet$ Miguel Montes) Uniciencia is protected by AttributionNonCommercial-NoDerivs 3.0 Unported (CC BY-NC-ND 3.0) 